\title{
EVALUATION OF GENE XPERT ASSAY IN EXTRAPULMONARY TUBERCULOSIS IN CHILDREN
}

\author{
Jitendra Singh Rathour1, Mukta Mantan², Ashwani Khanna³, Mohammed Hanif4 \\ 1MD, Department of Paediatrics, Maulana Azad Medical College, New Delhi, India. \\ 2Professor, Department of Paediatrics, Maulana Azad Medical College, New Delhi, India. \\ ${ }^{3}$ Chief Medical Officer, TB and Chest Hospital, Lok Nayak Hospital, New Delhi, India. \\ ${ }^{4}$ Bacteriologist, Laboratory Division, New Delhi Tuberculosis Center, New Delhi, India.
}

\begin{tabular}{l}
\hline ABSTRACT \\
BACKGROUND \\
Tuberculosis constitutes the main infectious cause of death worldwide. Extra-pulmonary tuberculosis (EPTB) accounts for up to \\
sixty percent of all cases of childhood tuberculosis. In high burden countries like India, WHO in December 2010 endorsed a new \\
rapid test "Gene Xpert MTB/RIF" as a replacement of sputum smear microscopy particularly in smear negative and multidrug \\
resistant TB. We aimed to assess the performance of the Xpert system in diagnosing EPTB in children in a high incidence settings \\
like India and conducted the present study at a tertiary care teaching hospital in New Delhi, India.
\end{tabular}

\section{MATERIALS AND METHODS}

This is a cross-sectional, hospital-based study. We investigated 60 extrapulmonary clinical specimens with Gene Xpert including 27 (45\%) cases of tubercular meningitis, 17 (28\%) cases of pleural tuberculosis, 12 (20\%) cases of tubercular lymphadenitis, 3 (5\%) cases of abdominal Koch's and one (2\%) case of pericardial tuberculosis and determined the sensitivity, specificity, positive predictive value (PPV) and negative predictive value (NPV) of CBNAAT and compared the efficacy of CBNAAT in the rate of detection of Mycobacteria, with the gold standard i.e. Culture (MGIT liquid culture).

\section{RESULTS}

The overall sensitivity of Xpert MTB/RIF was between 50 to 90 percent in various tissue samples while specificity in all samples was approaching almost 100 percent with maximum efficacy in tubercular lymphadenitis where the sensitivity was 90 percent followed by tubercular meningitis with 80 percent sensitivity.

\section{CONCLUSION}

Xpert MTB/RIF was found to be superior in detecting Mycobacterium tuberculosis in Extrapulmonary TB samples with respect to time of result and Rifampicin resistance detection. At present, CBNAAT is being used as a routine test in a suspected Extrapulmonary TB case at our center.

\section{KEY WORDS}

Gene Xpert MTB/RIF, MDR TB, Acid Fast Bacilli, MGIT Culture.

HOW TO CITE THIS ARTICLE: Rathour JS, Mantan M, Khanna A, et al. Evaluation of GENE XPERT assay in extrapulmonary tuberculosis in children. J. Evolution Med. Dent. Sci. 2019;8(01):76-80, DOI: 10.14260/jemds/2019/17

\section{BACKGROUND}

An estimated 1.3 million cases of tuberculosis and 450,000 associated deaths occur annually in children.(1) India has the world's largest burden of tuberculosis (TB), accounting for one-fourth $(24 \%)$ of the global TB incidence. The global annual incidence estimate is 9.9 million cases, of which 1.98 million cases are from India.(2) Among all cases of childhood tuberculosis, up to $60 \%$ cases are of Extrapulmonary tuberculosis.(3) The major challenge in the diagnosis of EPTB in children is the frequently atypical clinical presentation simulating other inflammatory and neoplastic conditions, which frequently results in a delay or deprivation of treatment.

'Financial or Other Competing Interest': None.

Submission 24-10-2018, Peer Review 25-12-2018,

Acceptance 31-12-2018, Published 07-01-2019.

Corresponding Author:

Dr. Jitendra Singh Rathour,

MD, Department of Paediatrics,

Maulana Azad Medical College,

Bahadur Shah Zafar Marg,

New Delhi-110002,

India

E-mail: drjeeturathour@gmail.com

DOI: $10.14260 /$ jemds/2019/17

\section{(c) (1) $(9)$}

Traditional smear microscopy is the only laboratory test for TB that is accessible to world's most of the population. Smear microscopy is inexpensive, appropriate for basic laboratories, rapidly diagnoses the most infectious patients and has high specificity so positive results almost always prompt the treatment. However, smear microscopy has two inadequacies-

1. It is insensitive, prone to false negative "Smear-negative TB" results.

2. It cannot test for drug resistance.

Which is important because patients with drug-resistant TB require prompt second line treatment to prevent morbidity, mortality, and dissemination of increasingly resistant multi-drug-resistant tuberculosis (MDR-TB) and extensively resistant TB (XDR-TB).

The diagnosis of extra pulmonary TB cases is challenging also due to inadequate clinical sample volumes available and paucibacillary nature of the biological samples. Patients with EPTB are more likely to have negative sputum smear results and many EPTB cases do not have direct lung involvement.

TB culture for diagnosing smear negative TB and testing for drug resistance takes considerable time (in weeks), too slow to address these adequacies. 


\section{Xpert MTB/RIF (CBNAAT: Cartridge Based Nucleic Acid Amplification Test)}

Xpert MTB/RIF is an automated polymerase chain reaction (PCR) test (molecular test) utilizing the Gene Xpert platform. Xpert MTB/RIF is a single test that can detect both $M$. tuberculosis complex and rifampicin resistance within two hours. Unlike conventional nucleic acid amplification tests (NAATs), Xpert MTB/RIF is unique because sample processing and PCR amplification and detection are integrated into a single self-enclosed test unit, the Xpert MTB/RIF cartridge. Following sample loading, all steps in the assay are completely automated and self-contained. In addition, the assay's sample reagent, used to liquefy the EPTB sample, has potent tuberculocidal (the ability to kill TB bacteria) properties and this largely eliminates biosafety concerns during the test procedure. The test material is combined with the assay sample reagent, mixed by hand or vortex, and incubated at room temperature for 15 minutes. After the incubation step, $2 \mathrm{ml}$ of the treated sample are transferred to the cartridge and the run initiated.

Xpert MTB/RIF uses molecular beacon technology to detect rifampicin resistance.(4) Molecular beacons are nucleic acid probes that recognize and report the presence or absence of the normal rifampicin susceptible, 'wild type' sequence of the rpoBgene of TB. Five different coloured beacons are used, each covering a separate nucleic acid sequence within the amplified rpoB gene. When a beacon binds to the matching sequence, it fluoresces or 'lights up', which indicates the presence of one of the gene sequences that is characteristic of rifampicin-susceptible TB.(5)

The number and timing of detection of positive beacons (When the fluorescent signal rises above a pre-determined baseline cycle threshold) as well as results of sample processing controls allow the test to distinguish among the following results: 'No TB'; 'TB detected, rifampicin resistance detected'; 'TB detected, no rifampicin resistance detected'; and an Invalid result'.

\section{Objectives \\ Primary Objective}

Rate of detection of Mycobacteria by Xpert assay in clinically suspected case of extrapulmonary tuberculosis

\section{Secondary Objective}

Comparing the rate of detection of Mycobacteria by Xpert assay in relation to smear positive and culture positive cases of Extrapulmonary TB

\section{MATERIALS AND METHODS}

We enrolled a total of 60 Children between the age group 0 14 years admitted in our paediatric wards or visiting the outpatient departments who fulfilled the inclusion criteria for Extrapulmonary TB. Out of 60 children, 27 (45\%) cases were of tubercular meningitis, 17 (28\%) cases were of pleural tuberculosis, 12 (20\%) cases were tubercular lymphadenitis, $3(5 \%)$ cases of abdominal Kochs while one (2\%) case was of pericardial tuberculosis.

Written Informed consent was taken from the parents of the children, also assent was taken from children $>7$ years of age. A detailed history was taken from the parents and a thorough physical examination was done for every patient.
Following tests were performed on each enrolled child-

1. Complete Blood Count with Erythrocytic sedimentation rate (ESR)

2. Mantoux test: Administration of 1 Tuberculin unit (TU) PPD intradermally, into the flexor aspects of left forearm, 2-4 inches below the elbow and the test was read after 48-72 hrs. Induration was measured in plane perpendicular to the long axis of forearm. Induration $>10$ $\mathrm{mm}$ was taken as positive test

3. Chest X-ray: - Every child underwent Chest X-ray to document the presence of Hilar lymphadenopathy with or without parenchymal changes, Miliary TB and Fibrocavitary lesion which have been considered highly suggestive of co-existing Pulmonary Tuberculosis.

History of Bacillus-Calmette-Guerin (BCG) immunization and Mantoux Positivity were also assessed in all 60 patients. Only 29 patients has received BCG immunization. On the other hand, 40 patients had positive Mantoux reaction. Specific investigations which were carried out to support the diagnosis of Different forms of EPTB shown various results as follows Table.

\begin{tabular}{|c|c|c|}
\hline $\begin{array}{c}\text { EPTB } \\
\text { Form }\end{array}$ & $\begin{array}{c}\text { Specific } \\
\text { Investigation }\end{array}$ & Result \\
\hline $\begin{array}{c}\text { TBM } \\
(\mathrm{n}=27)\end{array}$ & MRI-Brain & $\begin{array}{c}\text { Leptomeningeal enhancement } \\
\text { in all }(100 \%), \text { Basal exudate in } \\
24(88.8 \%), \text { Hydrocephalus in } \\
10(37.0 \%), \text { Tuberculoma in } \\
5(18.5 \%) .\end{array}$ \\
\hline $\begin{array}{c}\text { TBLN } \\
(\mathrm{n}=12)\end{array}$ & FNAC of & $\begin{array}{c}\text { Epithelial Cell granuloma in 10 } \\
(83.3 \%), \text { Acid -Fast bacilli in 4 } \\
(33.3 \%) \text { and caseous necrosis in } \\
\text { involved LN }(33.3 \%)\end{array}$ \\
\hline $\begin{array}{c}\text { AbTB } \\
(\mathrm{n}=3)\end{array}$ & CECT-Abdomen & $\begin{array}{c}\text { All three had mesenteric } \\
\text { lymphadenopathy, ascites and } \\
\text { bowel wall thickening. }\end{array}$ \\
\hline $\begin{array}{c}\text { PTB } \\
(\mathrm{n}=17)\end{array}$ & Chest X-ray & $\begin{array}{c}16 \text { patients had Unilateral } \\
\text { [Right (9) > Left (7)] pleural } \\
\text { effusion while one patient had } \\
\text { Bilateral pleural effusion }\end{array}$ \\
\hline $\begin{array}{c}\text { Pericardial } \\
\text { TB (n=1) }\end{array}$ & Echocardiography & $\begin{array}{c}\text { Moderate pericardial effusion } \\
\text { with fibrinous exudate }\end{array}$ \\
\hline \multicolumn{2}{|c|}{ Table 1 } \\
\hline
\end{tabular}

\section{Extrapulmonary TB Samples}

In our study, extrapulmonary samples were obtained during the clinical routine either from admitted children or on OPD basis and sent to Microbiology laboratory, Loknayak hospital for routine microscopy and New Delhi Tuberculosis (NDTB) Centre for CBNAAT result, between March 2014 to June 2015. Culture of extrapulmonary samples was done in NDTB Centre using Multiple Growth Indicator tube (MGIT) method.

Approximately $2.5 \mathrm{ml}$ sample was considered sufficient for CBNAAT processing. Following samples were obtained from various sites of extrapulmonary TB by prescribed methods-

1. Cerebrospinal fluid in case of TBM: The sample was obtained by Lumbar puncture technique.

2. Pleural fluid in case of Pleural TB: It was obtained by pleural tap by inserting needle in 7th-9th Intercostal space in scapular or posterior-axillary line.

3. Ascitic fluid in Abdominal Kochs: The needle was inserted into abdomen at the junction of lateral $1 / 3 \mathrm{rd}$ 
and medial $2 / 3 \mathrm{rd}$ of a line joining umbilicus to anterior superior iliac spine and fluid was aspirated.

4. Lymph abscess aspirate in Tubercular lymphadenitis: By simple aspiration with needle.

5. Pericardial aspirate in Pericardial TB by Pericardiocentesis by inserting the needle in 5th or 6th intercostal space at the left sternal border at the cardiac notch of the left lung under ultrasound guidance.

6. Joint aspirate in TB arthritis: Simple needle aspiration of joint fluid.

7. Gastric Aspirate (GA): Gastric aspirate from every children was taken on two consecutive days after overnight fast. This was obtained by inserting Nasogastric tube into the stomach and applying suction.

\section{Collection and Transport}

All of the above samples were sent to laboratory immediately after collection in sterile containers and proper labeling.Two vials containing EPTB specimens were sent to the New Delhi Tuberculosis (NDTB) Centre, one for CBNAAT and one part for MGIT Culture. There samples used to undergo decontamination and centrifugation. The pellet was then subjected to Ziehl-Neelsen (ZN) staining for Acid-Fast Bacilli (AFB).

One part each was sent to Microbiology laboratory of Lok Nayak Hospital for routine Microscopic examination, Sugar and protein estimation and culture-sensitivity.

\section{CBNAAT}

Sample reaching the NDTB center was processed as follows:

1. The sample reagent (Cepheid) was mixed at a 2:1 ratio with $1 \mathrm{ml}$ of the processed specimen.

2. The mixture used to be shaken and allowed to stand for five minutes.

3. The mixture then shaken again and allowed to stand for another 10 minutes.

4. Two millilitres of this homogenized mixture transferred into an CBNAAT: Xpert MTB/RIF assay cartridge and inserted into the Xpert instrument.

5. All subsequent steps were done automatically. 1 hour 45 minutes later, a printable result was obtained, showing CBNAAT positivity or negativity and presence or absence of Rifampicin resistance.

\section{Patient Groups}

Patients were grouped as

1. Those with smear- and culture positive tuberculosis;

2. Those with smear-negative, culture-positive tuberculosis

3. Those with no bacteriological evidence of tuberculosis.

\section{Statistical Analysis}

Descriptive analysis was done. Fisher's exact test was used to compare qualitative variables. To determine the means of quantitative variables, t-test was used. Sensitivity, specificity, Positive predictive value (PPV) and Negative predictive value (NPV) was calculated taking culture as Gold standard and analysis of categorical variables was examined by the chisquare test. A value of $\mathrm{P}$ of $<0.05$ was considered significant for all statistical analyses.

Data analysis was carried out with a statistical software package (SPSS) version 16.0

\section{RESULTS}

\section{Patients}

Sixty Extrapulmonary specimens from 60 children with mean age 7 years (Age ranging from 1 month to 14 years) with no sex predilection were included in the study.

\section{CBNAAT Positivity in Extrapulmonary Tissue/Fluid}

CBNAAT detected Tubercular bacterial DNA in different extrapulmonary specimen as follows:

Among 27 suspects of TBM, CBNAAT detected M.tuberculosis DNA in Cerebrospinal fluid (CSF) of 13 samples (48.1\%). In 12 TBLN suspects, CBNAAT was positive in 10 specimens of Lymph node aspirate (83.3\%). CBNAAT positivity was, however, comparatively low in Pleural TB Suspects i.e only 4 out of 17 (23.5\%) samples of Pleural fluid turned out to be positive for MTB-DNA. Among three cases of Abdominal TB, Ascitic fluid of 1 patient was CBNAAT Positive. The only patient of Pericardial TB was negative for CBNAAT Positive, but ADA level was raised in Pericardial fluid and the patient responded well to Anti-Tubercular Treatment (ATT).Table 2.

\begin{tabular}{|c|c|c|c|}
\hline $\begin{array}{r}\text { Sl. } \\
\text { No. }\end{array}$ & $\begin{array}{c}\text { EPTB } \\
\text { Tissue/Fluid }\end{array}$ & $\begin{array}{c}\text { CBNAAT } \\
\text { Positive }\end{array}$ & Percentage \\
\hline 1. & Cerebrospinal Fluid & $13 / 27$ & 48.1 \\
\hline 2. & Lymph Node Aspirate & $10 / 12$ & 83.3 \\
\hline 3. & Pleural Effusion & $6 / 17$ & 23.5 \\
\hline 4. & Ascitic Fluid & $1 / 3$ & 33.3 \\
\hline 5. & Pericardial Fluid & $0 / 1$ & 0 \\
\hline 6. & Gastric Aspirate & $21 / 60$ & $35 \%$ \\
\hline \multicolumn{3}{|c|}{ Table 2. CBNAAT Positivity of EPTB Tissue/Fluid } \\
\hline
\end{tabular}

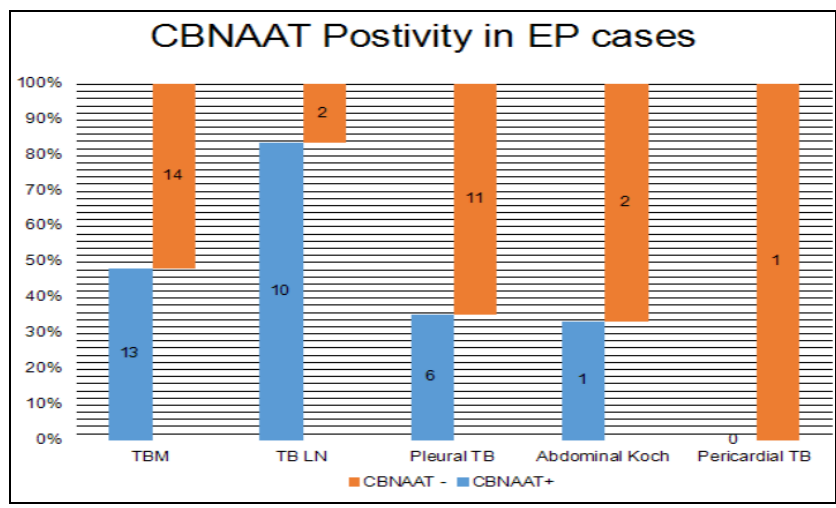

Culture Positivity of EPTB Specimens (Table 3)

1. TBM: 5 out of $27(18.5 \%)$ CSF samples grown $M$. tuberculosis on MGIT Liquid culture.

2. TBLN: 4 out of $12(33.3 \%)$ Lymph node aspirate samples were culture-positive.

3. PTB: 3 out of $17(17.6 \%)$ pleural fluid samples were culture - positive.

4. Abdominal TB: 2 ascitic fluid sample out of 3 (66.6\%) shown culture-positivity.

5. The only patient of Pericardial TB was culture-negative.

\begin{tabular}{|c|c|c|c|}
\hline $\begin{array}{c}\text { Sl. } \\
\text { No. }\end{array}$ & EPTB Tissue/Fluid & $\begin{array}{c}\text { Culture- } \\
\text { Positive }\end{array}$ & Percentage \\
\hline 1. & Cerebrospinal Fluid & $5 / 27$ & 18.5 \\
\hline
\end{tabular}




\begin{tabular}{|c|c|c|c|}
\hline 2. & Lymph Node Aspirate & $4 / 12$ & 33.3 \\
\hline 3. & Pleural Fluid & $3 / 17$ & 17.6 \\
\hline 4. & Ascitic Fluid & $2 / 3$ & 66.6 \\
\hline 5. & Pericardial Fluid & $0 / 1$ & 0 \\
\hline \multicolumn{3}{|c|}{ Table 3. Culture Positivity of EPTB Specimen } \\
\hline
\end{tabular}

After analysing the data of our study, CBNAAT had highest sensitivity in tubercular lymphadenitis i.e. $90.0 \%$ followed by tubercular meningitis in which CBNAAT had sensitivity of $54.5 \%$, while in abdominal Kochs, the sensitivity was $50.0 \%$. CBNAAT sensitivity in pleural TB was $41.6 \%$.

Sensitivity, Specificity, Positive Predictive Value \& Negative Predictive Value of CBNAAT in Relation to Culture

We calculated sensitivity, specificity, PPV and NPV in various extrapulmonary samples against the MGIT culture as Gold standard.

\section{Tubercular Lymphadenitis}

\begin{tabular}{|c|c|c|c|}
\hline \multirow{2}{*}{$\begin{array}{l}\text { Lymph Node } \\
\text { Aspirate }\end{array}$} & \multicolumn{2}{|c|}{ MGIT Liquid Culture } & \multirow{2}{*}{ Total } \\
\hline & Positive & Negative & \\
\hline Positive & 3 & 1 & 4 \\
\hline CDINAAI Negative & 1 & 7 & 8 \\
\hline Total & 4 & 8 & 12 \\
\hline $\begin{array}{l}\text { Sensiti } \\
\text { Specifi }\end{array}$ & $\begin{array}{r}\mathrm{ty}=75.0 \% \\
\mathrm{ty}=87.5 \% \\
\mathrm{PPV}= \\
\mathrm{NPV}=\end{array}$ & $\begin{array}{l}5 \% \text { CI } 21 . \\
5 \% \text { CI } 46.6 \\
0 \% \\
5 \%\end{array}$ & \\
\hline
\end{tabular}

\section{Tubercular Meningitis}

\begin{tabular}{|c|c|c|c|}
\hline \multirow{2}{*}{\begin{tabular}{|c|}
$\begin{array}{c}\text { Cerebrospinal } \\
\text { Fluid }\end{array}$ \\
\end{tabular}} & \multicolumn{2}{|c|}{ MGIT Liquid Culture } & \multirow{2}{*}{ Total } \\
\hline & Positive & Negative & \\
\hline Positive & 4 & 3 & 7 \\
\hline CBNAAT Negative & 1 & 19 & 20 \\
\hline Total & 5 & 22 & 27 \\
\hline \multicolumn{4}{|c|}{ Sensitivity $=80.0 \%$ (95\% CI 29.8-98.9) } \\
\hline \multicolumn{4}{|c|}{ Specificity $=86.3 \%(95 \%$ CI 64.0-96.4) } \\
\hline \multicolumn{4}{|c|}{$\mathrm{PPV}=57.1 \%$} \\
\hline \multicolumn{4}{|c|}{$\mathrm{NPV}=95.0 \%$} \\
\hline
\end{tabular}

\begin{tabular}{|lll|}
\hline \multicolumn{1}{|c}{$3)$} & \multicolumn{1}{c|}{ Pleural TB } & \multicolumn{1}{c|}{ Abdominal TB } \\
Sensitivity & $66.6 \%(95 \%$ Cl 12.5-98.2) & $50.0 \%(95 \%$ C12.6-97.3) \\
Specificity & $85.7 \%(95 \%$ Cl $56.1-97.4)$ & $100.0 \%(95 \%$ Cl $5.4-100.0)$ \\
PPV & $50.0 \%$ & $100.0 \%$ \\
NPV & $92.3 \%$ & $50.0 \%$ \\
\hline
\end{tabular}

\section{Rifampicin Resistance}

A total of $8(13.3 \%)$ EPTB suspects were found to have Rifampicin resistance on CBNAAT, out of which 5 cases were of tubercular meningitis, 2 cases of tubercular lymphadenitis while 1 case was of pleural tuberculosis. Prjapati S et al found a 5.5\% prevalence of Rifampicin resistance in Indian children with intrathoracic tuberculosis.(5)

\section{DISCUSSION}

Although the utility of CBNAAT has been well characterized in Pulmonary tuberculosis (Sputum sample), there are hardly any data to prove its usefulness in extrapulmonary tuberculosis. In this study, the performance of the MTB/RIF assay with extrapulmonary specimens was investigated.

In our study, the MTB/RIF test detected 30 of 60 extrapulmonary specimens (10 of 12 lymph node aspirate specimens, 13 of 27 cerebrospinal fluid specimens, 6 of 17 pleural fluid specimens, 1 of 3 ascitic fluid specimens while 1 of 1 pericardial fluid specimen was CBNAAT negative in whom ADA level in pericardial fluid was raised in tuberculosis range and the patient responded well to antitubercular treatment (ATT). In two Lymph node aspirate specimens, CBNAAT was negative, clinic-pathological profile along with Mantoux positivity and history of Contact suggested the diagnosis of Tuberculosis and both patients responded well to ATT.

Among the patients of TBM, sensitivity and specificity of CBNAAT was $80.0 \%$ and $86.3 \%$ which was comparable to the result of South African study carried by Vinod Patel and Grant theron.(6) CBNAAT was negative in 14 patients of TBM, but ADA levels were raised above $60 \mathrm{IU} / \mathrm{L}$ suggestive of tuberculosis. All these patients shown clinical improvement after starting ATT.

Among 17 patients of Pleural TB, only 6 patients were found to have MTB DNA by CBNAAT while only 3 cases were culture-positive. This could be due to very low numbers of bacilli in pleural fluid or the presence of antinhibitory substance which inhibited the amplification of the $M$. tuberculosis genome without any effect on the internal control for pleural fluid samples. Similar results of Gene Xpert study on Pleural fluid were found by Richard Meldau et al in Cape Town, South Africa.(7) In five cases of pleural TB, who were negative for MTB DNA in both GA and Pleural fluid, ADA levels in Pleural fluid were raised in the range $>25 \mathrm{IU} / \mathrm{L}$, suggestive of tuberculosis and these responded well to ATT drugs.

Similarly, in one case of abdominal TB, ascitic fluid as well as GA were negative for CBNAAT but ADA level in ascetic fluid was above the diagnostic cut-off value ( $>40 \mathrm{IU} / \mathrm{L})$. Lesley Erica Scotta et al in their study on ascitic fluid found a matching result by Gene Xpert. (8)

\section{CONCLUSION}

The purpose of our study was to determine the efficacy of Gene Xpert MTB/RIF (CBNAAT) in extrapulmonary tuberculosis (EPTB) in children aged 0-14 years.

- CBNAAT was found to have maximum utility in diagnosing clinical cases of tubercular lymphadenitis with a sensitivity of $90.0 \%$ followed by tubercular meningitis with a sensitivity of $54.5 \%$, abdominal $\mathrm{TB}$ sensitivity $50.0 \%$ and pleural TB (sensitivity $41.6 \%$ ).

- When compared with culture, CBNAAT has highest sensitivity of detection of MTB DNA in cerebrospinal fluid with a sensitivity of $80.0 \%$ followed by tubercular lymphadenitis with a sensitivity of $75.0 \%$ and abdominal TB (sensitivity $50.0 \%$ ). The sensitivity of CBNAAT in detecting TB bacilli in pleural fluid was the least- $41.6 \%$.

- CBNAAT detected Rifampicin resistance in 8 cases (13.3\%), the maximum cases were of tubercular meningitis while 2 were of tubercular lymphadenitis while one was of pleural TB. All these were labelled as MDR TB and treated accordingly. All of them responded to treatment except one case of TBM who expired after 2 weeks of treatment. 
However, it is emphasized that MDR status must be confirmed with Drug susceptibility testing (DST).

The MTB/RIF test is less dependent on the user's skills, and routine staff with minimal training can use the test. It has a short turnaround time and simultaneously detects $\mathrm{M}$. tuberculosis and RIF resistance in less than $2 \mathrm{~h}$. Although the MTB/RIF test could be a useful tool for rapid identification of RIF-resistant M. tuberculosis, especially in smear-positive clinical samples, the test results must always be confirmed by culture and DST.

Main drawback of CBNAAT is its cost and requirement of infrastructure. Larger trials are needed to assess the exact efficacy of CBNAAT in extrapulmonary tuberculosis.

\section{Limitation of the Test}

The Xpert test is a major advance in TB diagnostic testing, but has few limitations such as the limited shelf-life of the diagnostic cartridges, some operating temperature and humidity restrictions, requirement for electricity supply, unknown long term robustness, and the need for annual servicing and calibration of each machine. Laboratories in low-income countries are littered with expensive equipment that no longer functions because it was inappropriate to the setting to which it was donated. Ensuring sustainable systems for long-term provision of servicing and consumables may be more important and challenging than initial implementation of the diagnostic equipment itself.

\section{Impact in Low- and Middle-Income Countries}

In spite of a number of studies of improved TB diagnostic technologies, enough guidelines of how best to implement better tests to improve patient care are still lacking. Nowhere it is clearly advised as to who should receive the better tests to maximize health impact. Assessment regarding how these tests may impact patient-relevant outcomes has to be done still. The impact of better diagnostic tests on the equity of care is largely unstudied and still yet to determine how this novel technology will affect the delays and costs faced by patients. (9)

\section{REFERENCES}

[1] Raviglione MC, Snider DE, Kochi A. Global epidemiology of tuberculosis. Morbidity and mortality of a worldwide epidemic. JAMA 1995;273(3):220-6.

[2] Raj A, Netrapal S, Pramod KM. Gene Xpert MTB/RIF assay: a new hope for extrapulmonary tuberculosis. IOSR Journal of Pharmacy 2012;2(1):83-9.

[3] Sivanandan S, Walia M, Lodha R, et al. Factors associated with treatment failure in childhood tuberculosis. Indian Pediatr 2008;45(9):769-71.

[4] El-Hajj HH, Marras SA, Tyagi S, et al. Detection of rifampin resistance in mycobacterium tuberculosis in a single tube with molecular beacons. J Clin Microbiol 2001;39(11):4131-7.

[5] Prajapati S, Upadhyay K, Mukherjee A, et al. High prevalence of primary drug resistance in children with intrathoracic tuberculosis in India. Paediatr Int Child Health 2016;36(3):214-8.

[6] Patel VB, Theron G, Lenders L, et al. Diagnostic accuracy of quantitative PCR (Xpert MTB/RIF) for tuberculous meningitis in a high burden setting: a prospective study. PLoS Med 2013;10(10):e1001536.

[7] Meldau R, Peter J, Theron G, et al. Comparison of same day diagnostic tools including Gene Xpert and unstimulated IFN- $\gamma$ for the evaluation of pleural tuberculosis: a prospective cohort study. BMC Pulmonary Medicine 2014;14:58.

[8] Scott LE, Beylis N, Nicol M, et al. Diagnostic accuracy of Xpert MTB/RIF for extrapulmonary tuberculosis specimens: establishing a laboratory testing algorithm for South Africa. J Clin Microbiol 2014;52(6):1818-23.

[9] Evans CA. GeneXpert--a game-changer for tuberculosis control? PLoS Med 2011;8(7):e1001064. 